\title{
The Representations of the $q$-analogue of Brauer's Centralizer Algebras and the Kauffman Polynomial of Links ${ }^{1}$
}

\author{
By
}

Jun Murakami*

\section{§0. Introduction}

Let $\mathbf{C}(\alpha, q)$ be the two-variable rational function field over $\mathbb{C}$ with indeterminates $\alpha$ and $q$. It is shown in [3] and [8] that the Kauffman polynomial of links are associated with a sequence of $\mathbf{C}(\alpha, q)$-algebras, which are denoted by $C_{n}(\alpha, q)(n \in \mathbb{N})$. These algebras can be considered as $q$-analogues of Brauer's algebras $D_{n}(\beta)$ [4] defined over $\mathbb{C}(\beta)$, where $\beta$ is an indeterminate. Let $G$ be a group of linear transformations on a vector space $V$ and $\pi^{\otimes n}$ the representation of $G$ on $V^{n}=V \otimes \cdots \otimes V$, the $n$-th tensor power of $V$. Let $Z_{n}(G)$ be the centralizer algebras of $\pi^{\otimes n}$, i.e.

$$
Z_{n}(G)=\left\{x \in \operatorname{End}\left(V^{n}\right) \mid x \pi^{\otimes n}(g)=\pi^{\otimes n}(g) x \quad \text { for all } g \in G\right\} .
$$

Let $G$ be the symplectic group $S p_{2 m}$ or the special orthogonal group $S O_{2 m+1}$. Then $Z_{n}(G)$ is a semisimple quotient of the algebra $D_{n}(\beta)$. From the results of [3], [5], [8] and [10], we have an analogous result for $C_{n}(\alpha, q)$. Let $\mathfrak{g}$ be one of Lie algebras $\mathfrak{s p}_{2 m}$ and $\mathfrak{s o}_{2 m+1}$ of the Lie groups $S p_{2 m}$ and $S O_{2 m+1}$. Let $\hat{\mathscr{U}}(\mathrm{g})$ be the $q$-analogue of the universal enveloping algebra $\mathscr{U}(\mathrm{g})$ (see e.g. [5]). Then there is an integer $r$ such that the centralizer algebras associated with the vector representation of $\hat{\mathscr{U}}(\mathrm{g})$ are quotients of the algebras $C_{n}\left(q^{r}, q\right)$.

The aim of the present paper is to construct irreducible representations of $C_{n}(\alpha, q)$ explicitly. The $\mathbb{C}(\alpha, q)$-algebra $C_{n}(\alpha, q)(n \in \mathbb{N})$ with 1 is defined by the following.

Communicated by H. Araki, September 13, 1988. Revised February 5, 1990.

* Department of Mathematics, Osaka University, Toyonaka, Osaka 560, Japan.

1 This research was supported in part by Yukawa Foundation and Grand-in-Aid for Scientific Research, The Ministry of Education, Science and Culture. 


$$
\begin{aligned}
& C_{1}(\alpha, q)=\mathbb{C}(\alpha, q), \\
& C_{n}(\alpha, q)=\left\langle\tau_{i}, \tau_{i}^{-1}, \varepsilon_{i}(1 \leq i \leq n-1)\right| \tau_{i} \tau_{i+1} \tau_{i}=\tau_{i+1} \tau_{i} \tau_{i+1}, \\
& \quad \varepsilon_{i} \varepsilon_{i+1} \varepsilon_{i}=\varepsilon_{i}, \varepsilon_{i+1} \varepsilon_{i} \varepsilon_{i+1}=\varepsilon_{i+1}, \\
& \quad \tau_{i}^{ \pm 1} \varepsilon_{i+1} \varepsilon_{i}=\tau_{i+1}^{\mp 1} \varepsilon_{i}, \tau_{i+1}^{ \pm 1} \varepsilon_{i} \varepsilon_{i+1}=\tau_{i}^{\mp 1} \varepsilon_{i+1}, \\
& \quad \varepsilon_{i} \varepsilon_{i+1} \tau_{i}^{ \pm 1}=\varepsilon_{i} \tau_{i+1}^{\mp 1}, \varepsilon_{i+1} \varepsilon_{i} \tau_{i+1}^{ \pm 1}=\varepsilon_{i+1} \tau_{i}^{\mp 1}(1 \leq i \leq n-2), \\
& \tau_{i} \tau_{j}=\tau_{j} \tau_{i}, \varepsilon_{i} \tau_{j}=\tau_{j} \varepsilon_{i}, \varepsilon_{i} \varepsilon_{j}=\varepsilon_{j} \varepsilon_{i}(1 \leq i<j-1 \leq n-2), \\
& \tau_{i} \tau_{i}^{-1}=\tau_{i}^{-1} \tau_{i}=1, \tau_{i} \varepsilon_{i}=\varepsilon_{i} \tau_{i}=\left(-\alpha^{2} q\right)^{-1} \varepsilon_{i}, \\
& \left.\tau_{i}-\tau_{i}^{-1}=\left(q-q^{-1}\right)\left(1-\varepsilon_{i}\right)(1 \leq i \leq n-1)\right\rangle(n \geq 2) .
\end{aligned}
$$

Note that (0.1) implies that $\varepsilon_{i}^{2}=\left(1-\frac{\alpha^{2} q-\alpha^{-2} q^{-1}}{q-q^{-1}}\right) \varepsilon_{i}$. Hence the algebra $C_{n}(\alpha, q)$ is a one-parameter deformation of the algebra $D_{n}(\beta)$. More precisely, $D_{n}(\beta)$ is the limit $q \rightarrow 1$ of $C_{n}\left(q^{\beta / 2}, q\right)$. The algebra $D_{n}(\beta)$ is semisimple and its irreducible representations are classified [3]. Hence its one-parameter deformation $C_{n}(\alpha, q)$ is semisimple and there is a bijection between the irreducible representations of $C_{n}(\alpha, q)$ and those of $D_{n}(\beta)$. Let $\rho$ be an irreducible representation of $C_{n}(\alpha, q)$. Then the representation matrices of $\rho\left(\tau_{i}\right), \rho\left(\tau_{i}^{-1}\right)$ and $\rho\left(\varepsilon_{i}\right)(1 \leq i \leq n-1)$ with respect to a certain basis are given in $\S 1$. By taking the limit $q \rightarrow 1$ of the above matrices, we get the irreducible representations of the algebra $D_{n}(\beta)$. Our construction is based on [6].

Let $B_{n}$ denote the braid group on $n$-strings and $\sigma_{i}(1 \leq i \leq n-1)$ its standard generators of $B_{n}$. Let $p_{n}$ be the algebra homomorphism from the group ring $\mathbb{C} B_{n}$ to $C_{n}(\alpha, q)$ defined by $p_{n}\left(\sigma_{i}^{ \pm 1}\right)=\left(-\alpha^{2} q\right)^{\mp 1} \tau_{i}^{ \pm 1}(1 \leq i \leq n$ $-1)$. For $\rho \in C_{n}(\alpha, q)^{\wedge}$ let $\chi_{\rho}$ denote the character of $\rho \circ p_{n}$. For $b \in B_{n}$, let $\hat{b}$ denote the closure of $b$. Let $F(\hat{b})$ denote the Kauffman polynomial of the closed braid $\hat{b}$ of $b \in B_{n}$ with values in $C_{n}(\alpha, q)$. Then there are $a_{\rho} \in \mathbb{C}(\alpha, q)$ for $\rho \in C_{n}(\alpha, q)^{\wedge}$ such that

$$
F(\hat{b})=\sum_{\rho \in C_{n}(\alpha, q) \wedge} a_{\rho} \chi_{\rho}(b) \quad \text { (see [3] and [8]). }
$$

We explicitly give the coefficients $a_{\rho}$ in (2.1) and Theorem 2.2. Hence (0.2) can be used to calculate the Kauffman polynomial of closed braids. The representation matrices of $\rho\left(\tau_{i}\right)$ and $\rho\left(\tau_{i}^{-1}\right)(1 \leq i \leq n-1)$ given in $\S 1$ are all symmetric. This fact is used to show [9, Theorem 6.2.4], which claims the following. Let $v$ be a one-dimensional representation of $C_{n}(\alpha, q)$ and $F^{(r, v)}$ a link invariant associated with $F$ and $v$ introduced in [9, Section 1.5]. Then we have $F^{(r, v)}(K)=F^{(r, v)}\left(K^{\prime}\right)$ for mutant knots $K$ and $K^{\prime}$.

I would like to express my thanks to M. Jimbo and T. Miwa who gave me much information about solutions of Yang-Baxter equations, including the result of [6]. 


\section{§1. Construction}

Fix a positive integer $n$. Let

$$
\Lambda=\left\{\left(\lambda_{1}, \lambda_{2}, \ldots\right) \mid \lambda_{i} \geq \lambda_{i+1} \geq 0(i \in \mathbb{N}), \lambda_{j}=0(j \gg 0)\right\} .
$$

An element of $\Lambda$ is called a partition. We also use the following notation.

$$
\Lambda(n)=\left\{\left(\lambda_{1}, \lambda_{2}, \ldots\right) \in \Lambda \mid \sum_{i \in \mathbf{N}} \lambda_{i}=n-2 j, 0 \leq j \leq\left[\begin{array}{c}
n \\
2
\end{array}\right]\right\} .
$$

For two partitions $\lambda=\left(\lambda_{1}, \lambda_{2}, \ldots\right)$ and $\lambda^{\prime}=\left(\lambda_{1}^{\prime}, \lambda_{2}^{\prime}, \ldots\right)$ in $\Lambda$, we denote $\lambda \sim \lambda^{\prime}$ if there is $j \in \mathbb{N}$ such that $\lambda_{i}=\lambda_{i}^{\prime}$ for $i \neq j$ and $\lambda_{j}=\lambda_{j}^{\prime} \pm 1$. For a partition $\lambda \in \Lambda(n)$, let

$$
\begin{aligned}
& \mathscr{P}(\lambda)=\left\{P=\left(\lambda^{(0)}, \lambda^{(1)}, \ldots, \lambda^{(n)}\right) \mid \lambda^{(0)}\right.=(0,0, \ldots), \lambda^{(n)}=\lambda, \\
&\left.\lambda^{(i)} \sim \lambda^{(i+1)} \text { for } 0 \leq i \leq n-1\right\} .
\end{aligned}
$$

Let $V_{\lambda}=\oplus_{P \in \mathscr{P}(\lambda)} \mathbb{C}(\alpha, q) v_{P}$, which is a vector space over $\mathbb{C}(\alpha, q)$ with a basis $\left\{v_{P} \mid P \in \mathscr{P}(\lambda)\right\}$. In this paper, we use the following notations.

$$
\{k\}=\frac{q^{k}-q^{-k}}{q-q^{-1}}, \quad\{k ; m\}=\frac{\alpha^{m} q^{k}-\alpha^{-m} q^{-k}}{q-q^{-1}} .
$$

For $v=\left(v_{1}, v_{2}, \ldots, v_{\ell}, 0,0, \ldots\right) \in \Lambda$, let

$$
\begin{aligned}
& h_{v}(i, j)= v_{i}-i-j+\max \left\{k \mid v_{k} \geq j\right\}\left(1 \leq i \leq \ell, 1 \leq j \leq v_{i}\right), \\
& g_{v}(i)=\left\{\begin{array}{l}
\frac{\prod_{j=i}^{v_{i}+i-1}\left\{v_{i}+v_{j}+2-i-j ; 2\right\}}{\prod_{j=1}^{v_{i}}\{j-i+1 ; 1\}}\left(v_{i}+i>\ell\right), \\
\frac{\prod_{j=i}^{\ell}\left\{v_{i}+v_{j}+2-i-j ; 2\right\}}{\prod_{j=1}^{v_{i}}\{j-i+1 ; 1\} \prod_{j=1}^{\ell-v_{i}-i+1}\{3-2 i-j ; 2\}}\left(v_{i}+i \leq \ell\right),
\end{array}\right.
\end{aligned}
$$

and

$$
G_{v}=\prod_{i=1}^{\ell} g_{v}(i)\left(\prod_{j=1}^{v_{2}} \frac{\{j-i ; 1\}}{\left\{h_{v}(i, j)+1\right\}}\right) .
$$

Fix $i$ in $\{1,2, \ldots, n-1\}$. We define an element $A_{i}$ in End $\left(V_{\lambda}\right)$. We give the matrix of $A_{i}$ with respect to the basis $\left\{v_{P} \mid P \in \mathscr{P}(\lambda)\right\}$. Let $P=\left(\lambda^{(0)}, \lambda^{(1)}, \ldots, \lambda^{(n)}\right)$ and $Q=\left(v^{(0)}, v^{(1)}, \ldots, v^{(n)}\right)$ be elements of $\mathscr{P}(\lambda)$. The elements of the partition $\lambda^{(r)}$ is denoted by $\lambda_{1}^{(r)}, \lambda_{2}^{(r)}, \ldots$. Let $\ell$ denote the maximal integer satisfying $\lambda_{\ell}^{(i-1)} \neq 0$ and $\left(\eta_{1}, \ldots, \eta_{\ell}, 0, \ldots\right)=\lambda^{(i-1)}$. We put

$$
\eta(i)=\eta_{i}-i+1
$$


Let $A_{i} v_{P}=\sum_{Q \in \mathscr{P}(\lambda)}\left(A_{i}\right)_{Q P} v_{Q}$. In the following, $\delta_{1}$ and $\delta_{2}$ denote either 1 or - 1. If there is an element $j \in\{1,2, \ldots, n-1\} \backslash\{i\}$ such that $\lambda^{(j)} \neq v^{(j)}$, then we put

$$
\left(A_{i}\right)_{Q P}=0
$$

From now on, we treat the case that $\lambda^{(j)}=v^{(j)}$ for $j \in\{1,2, \ldots, n-1\} \backslash\{i\}$. At first we assume that $P=Q$. If there is an $r$ in $\mathbb{N}$ such that $\lambda_{r}^{(i-1)}=\lambda_{r}^{(i+1)} \pm 2$, then we put

$$
\left(A_{i}\right)_{P P}=q .
$$

If $\lambda^{(i-1)}=\lambda^{(i+1)}$, then there is a unique $r$ such that $\lambda_{r}^{(i-1)}=\lambda_{r}^{(i)}-\delta_{1}$. For such $P$, we put

$$
\left(A_{i}\right)_{P P}=-\frac{\alpha^{-2 \delta_{1}} q^{-2 \delta_{1} \eta(r)-1}}{\left\{2 \delta_{1} \eta(r)+1 ; 2 \delta_{1}\right\}}-\frac{\alpha^{-2 \delta_{1}} q^{-2 \delta_{1} \eta(r)-1} G_{\lambda^{(2)}}}{G_{\lambda^{(i-1)}}\left\{2 \delta_{1} \eta(r)+1 ; 2 \delta_{1}\right\}} .
$$

If otherwise, there are unique $r$ and $s$ in $\mathbb{N}$ such that $r \neq s, \lambda_{r}^{(i-1)}=\lambda_{r}^{(i)}-\delta_{1}$ and $\lambda_{s}^{(i)}=\lambda_{s}^{(i+1)}-\delta_{2}$. For such $P$, we put

$$
\left(A_{i}\right)_{P P}=-\frac{\alpha^{\delta_{2}-\delta_{1}} q^{\delta_{2} \eta(s)-\delta_{1} \eta(r)}}{\left\{\delta_{1} \eta(r)-\delta_{2} \eta(s) ; \delta_{1}-\delta_{2}\right\}} .
$$

Now, we assume that $P \neq Q$. If $\lambda^{(i-1)} \neq \lambda^{(i+1)}$, then there are unique $r$ and $s$ in $\mathbb{N}$ such that $r \neq s$ and $\lambda_{r}^{(i-1)}=\lambda_{r}^{(i)}-\delta_{1}, \lambda_{s}^{(i)}=\lambda_{s}^{(i+1)}-\delta_{2}$. For such $P$ and $Q$, we put

$$
\left(A_{i}\right)_{Q P}=\frac{\sqrt{\left\{\delta_{1} \eta(r)-\delta_{2} \eta(s)+1 ; \delta_{1}-\delta_{2}\right\}\left\{\delta_{1} \eta(r)-\delta_{2} \eta(s)-1 ; \delta_{1}-\delta_{2}\right\}}}{\left\{\delta_{1} \eta(r)-\delta_{2} \eta(s) ; \delta_{1}-\delta_{2}\right\}}
$$

If $\lambda^{(i-1)}=\lambda^{(i+1)}$ then there are unique $r$ and $s$ in $\mathbb{N}$ such that $\lambda_{r}^{(i-1)}=\lambda_{r}^{(i)}-\delta_{1}$ and $v_{s}^{(i-1)}=v_{s}^{(i)}-\delta_{2}$. For such $P$ and $Q$, we put

$$
\left(A_{i}\right)_{Q P}=-\frac{\alpha^{-\delta_{1}-\delta_{2}} q^{-\delta_{1} \eta(r)-\delta_{2} \eta(s)-1} \sqrt{G_{\lambda^{(l)}} G_{v^{(2)}}}}{G_{\lambda^{(1-1)}}\left\{\delta_{1} \eta(r)+\delta_{2} \eta(s)+1 ; \delta_{1}+\delta_{2}\right\}} .
$$

The above definition of $A_{i}$ implies that $\left(A_{i}\right)_{Q P}=\left(A_{i}\right)_{P Q}$ for $P, Q \in \mathscr{P}(\lambda)$, in other words, $A_{i}(1 \leq i \leq n-1)$ are symmetric matrices. Let $P=\left(\lambda^{(0)}, \lambda^{(1)}, \ldots, \lambda^{(n)}\right)$ and $Q=\left(v^{(0)}, v^{(1)}, \ldots, v^{(n)}\right) \in \mathscr{P}(\lambda)$ such that $\lambda^{(j)}=v^{(j)}$ for $j \in\{1,2, \ldots, n\} \backslash\{i\}$. By (1.2), the matrix element $\left(A_{i}\right)_{Q P}$ does not depend on $\lambda^{(j)}, j \neq i, i \pm 1$. Let $A\left(\lambda^{(i-1)}, \lambda^{(i)}, v^{(i)}, \lambda^{(i+1)}\right)=\left(A_{i}\right)_{Q P}$. Let $W_{m}^{t}\left(\begin{array}{ll}a & b \\ d & c\end{array} \mid u\right)$ denote the trigonometric limit of the Boltzmann weight defined in [6] associated with the Lie algebra of Dynkin type $C_{m}^{(1)}$. In this case, the function $[u]$ used in the definition of the Boltzmann weight in [6] is equal to $2 \sin (\pi u / L)$, where $L$ is an arbitrary non- 
zero complex parameter. Put $x=\exp (\pi u \sqrt{-1} / L)$. Recall that $W_{m}^{t}\left(\begin{array}{ll}a & b \\ d & c\end{array} \mid u\right)$ is a Laurent polynomial in $x$ and the highest degree of $W_{m}^{t}\left(\begin{array}{ll}a & b \\ d & c\end{array} \mid u\right)$ with respect to $x$ is equal to $2((1.5)$ of [6]). For dominant integral weight $a$ of the simple Lie algebra of Dynkin type $C_{m}$ given as in Table 1 of [6], let $\lambda(a)$ $=\left(\lambda_{1}, \lambda_{2}, \ldots, \lambda_{n}, 0, \ldots\right)$ be an element of $\Lambda$ such that

$$
\lambda_{i}=a_{i}-n-1+i \text {. }
$$

By comparing the definition of $A_{i}$ and (1.5) of [6], we have the following.

Lemma 1.4. For dominant integral weights $a, b, c$ and $d$ of the simple Lie algebra of Dynkin type $C_{m}$ such that $\lambda(a) \sim \lambda(b), \lambda(b) \underset{1}{\sim} \lambda(c), \lambda(c) \tilde{\tau} \lambda(d)$ and $\lambda(d) \sim_{1} \lambda(a)$, we have

$$
\lim _{u \rightarrow \infty} W_{m}^{t}\left(\begin{array}{ll}
a & b \\
d & c
\end{array} \mid-\sqrt{-1} u\right) x^{-2}=\left.\lim _{q \rightarrow e^{\sqrt{-1}} \pi / L} \frac{q^{(m+1)} A(\lambda(a), \lambda(b), \lambda(d), \lambda(c))}{\{m+1\}\{1\}^{2}}\right|_{\alpha=q^{m}},
$$

where $L$ is the complex parameter used in [6].

Proof. For a dominant integral weight $a=\left(a_{1}, a_{2}, \ldots, a_{m}\right)$, let

$$
G_{a}^{\prime}=\varepsilon(a) \prod_{j=1}^{m}\left[2 a_{j}\right] \prod_{1 \leq j<k \leq m}\left[a_{j}-a_{k}\right]\left[a_{j}+a_{k}\right](\varepsilon(a)= \pm 1) .
$$

Then the above lemma is proved by using the fact that the limit of $\left.G_{\lambda(a)}\right|_{\alpha=q^{m}}$ with $q$ to $e^{\sqrt{-1} \pi / L}$ is equal to the trigonometric limit of $\varepsilon(a) G_{a(v)}^{\prime}\left(\prod_{1 \leq j<k \leq m}[i\right.$ $-k])^{-1}\left(\prod_{1 \leq j \leq k \leq m}[i+k]\right)^{-1}$. We omit the detail.

The following theorem is the main result of this paper.

Theorem 1.5. Let $\lambda$ be a partition in $\Lambda(n)$.

(i) There is a representation $\left(\rho_{\lambda}, V_{\lambda}\right)$ of $C_{n}(\alpha, q)$ such that the representation matrices of $\rho_{\lambda}\left(\tau_{i}\right)$ is equal to $A_{i}$ defined by (1.2).

(ii) The representation $\rho_{\lambda}$ is irreducible.

(iii) Two irreducible representations $\rho_{\lambda}$ and $\rho_{v}(\lambda, v \in \Lambda(n), \lambda \neq v)$ are not equivalent.

(iv) Any irreducible representation of $C_{n}(\alpha, q)$ is equivalent to one of $\left(\rho_{\lambda}, V_{\lambda}\right)$ $(\lambda \in \Lambda(n))$.

A similar result holds for the Brauer's algebra $D_{n}(\beta)$.

Corollary 1.6. Any irreducible representation of $D_{n}(\beta)$ is a limit of $\left.\rho_{\lambda}\right|_{\alpha=q^{\beta / 2}}$ with $q \rightarrow 1$.

In the rest of this section we give proofs of the above theorem and its 
corollary. Some combinatorial consideration about partitions shows the following two lemmas.

Lemma 1.7. For $\lambda \in \Lambda(n)$, let $v$ and $v^{\prime}$ be two elements of $\Lambda(n-1)$ such that $v \sim \lambda$ and $v^{\prime} \sim \lambda$. Then there is $\mu \in \Lambda(n-2)$ such that $\mu \sim{ }_{1} v$ and $\mu \sim v^{\prime}$.

Lemma 1.8. For $v \in \Lambda(n)$, let $L(v)=\{\mu \in \Lambda(n-1) \mid \mu \sim v\}$. For $v_{1}, v_{2} \in \Lambda(n)$ with $n \geq 3$, assume that $L\left(v_{1}\right)=L\left(v_{2}\right)$. Then $v_{1}=v_{2}$.

We use the following to show the relation $E_{i}^{2}=\left(1-\left(\alpha^{2} q-\alpha^{-2} q^{-1}\right)(q\right.$ $\left.\left.-q^{-1}\right)^{-1}\right) E_{i}$.

Lemma 1.9. For $\lambda \in \Lambda$, we have

$$
\sum_{\nu \widetilde{1} \lambda} \frac{G_{v}}{G_{\lambda}}=\frac{\alpha^{2} q-\alpha^{-2} q^{-1}}{q-q^{-1}}-1 .
$$

Proof. It suffices to show in the case $\alpha=q^{m}$ for infinitely may integers $m$. Fix an integer $m \geq \sum_{i \in \mathbb{N}} \lambda_{i}$. We use representation theory of the Lie algebra $\mathfrak{g}$ of type $C_{m}$. Let $\Delta$ be a root system corresponding to $g$ and $\Gamma$ the weight lattice associated with $\Delta$. Fix a simple root system $\Pi$ in $\Delta$ and let $\Delta_{+}$be the set of positive roots associated with $\Pi$. Let $\psi=\sum_{\mu \in \Delta_{+}} \mu$. For a g-module $V$, we denote $m_{V}(\mu)$ the dimension of the weight space of $V$ corresponding to $\mu$. Let

$$
d_{q}(V)=\sum_{\mu \in \Gamma} m_{V}(\mu) q^{(\psi, \mu)}
$$

where (,) is the natural pairing of roots and weights. For $v \in \Lambda$, let $V_{v}$ be the simple $\mathfrak{g}$-module with highest weight corresponding to $v$ as in (1.3). By using the $q$-analogue of Weyl's character formula ([7], proof of Corollary 8.9), we have

$$
G_{v}=d_{q}\left(V_{v}\right)
$$

On the other hand, we know that $\oplus_{v \tau_{1} \lambda} V_{v}=V_{\lambda} \otimes V_{(1,0,0, \ldots)}$, where $V_{(1,0,0, \ldots)}$ is corresponding to the vector representation of $\mathfrak{g}$. Hence (1.10) and (1.11) imply $\sum_{v \tilde{i}_{\lambda}} G_{v}=G_{\lambda} G_{(1,0,0, \ldots)}$. Since $G_{(1,0,0, \ldots)}=1-\frac{q^{2 m+1}-q^{-2 m-1}}{q-q^{-1}}$, we get the statement of the lemma in the case $\alpha=q^{m}$.

Proof of Theorem 1.5. We first prove the statement (i). Let $A_{i}^{\prime}$ be the matrix obtained from $A_{i}$ by substituting $\alpha^{-1}$ to $\alpha$ and $q^{-1}$ to $q$. Then Lemma 1.4 and the inversion relation $[6,(2.13 \mathrm{a})]$ imply that $A_{i} A_{i}^{\prime}=1$. Let

$$
E_{i}=-\frac{A_{i}-A_{i}^{\prime}}{q-q^{-1}}+1 \text {. }
$$


Let $P=\left(\lambda^{(0)}, \lambda^{(1)}, \ldots, \lambda^{(n)}\right)$ and $Q=\left(v^{(0)}, v^{(1)}, \ldots, v^{(n)}\right)$ be two elements of $\mathscr{P}(\lambda)$. Then the matrix element $\left(E_{i}\right)_{Q P}$ is given as follows. If there is an element $j \in\{1,2, \ldots, n\} \backslash\{i\}$ such that $\lambda^{(j)} \neq v^{(j)}$, then we have

$$
\left(E_{i}\right)_{Q P}=0 \text {. }
$$

From now on, we treat the case that $\lambda^{(j)}=v^{(j)}$ for $j \in\{1,2, \ldots, n\} \backslash\{i\}$. If $\lambda^{(i-1)} \neq \lambda^{(i+1)}$, then we have

$$
\left(E_{i}\right)_{Q P}=0
$$

If $\lambda^{(i-1)}=\lambda^{(i+1)}$ and $P \neq Q$, then we have

$$
\left(E_{i}\right)_{Q P}=-\frac{\sqrt{G_{\lambda^{(2)}} G_{v^{(2)}}}}{G_{\lambda^{(i-1)}}} .
$$

If $\lambda^{(i-1)}=\lambda^{(i+1)}$ and $P=Q$, then there is an $r$ in $\mathbf{N}$ and $\delta_{1}= \pm 1$ such that $\lambda_{r}^{(i-1)}=\lambda_{r}^{(i)}-\delta_{1}$ and we have

$$
\left(E_{i}\right)_{P P}=-\frac{G_{\lambda^{(2)}}}{G_{\lambda^{(2-1)}}} .
$$

By using Lemma 1.9, the matrix $E_{i}$ satisfies

$$
E_{i}^{2}=-\left(\frac{\alpha^{2} q-\alpha^{-2} q^{-1}}{q-q^{-1}}-1\right) E_{i}
$$

We have relations

$$
E_{i} E_{i \pm 1} E_{i}=E_{i}
$$

from $(-1)^{3} \frac{\sqrt{G_{v_{1}} G_{v_{3}}}}{G_{v_{2}}} \frac{\sqrt{G_{v_{2}} G_{v_{2}}}}{G_{v_{3}}} \frac{\sqrt{G_{v_{3}} G_{v_{4}}}}{G_{v_{2}}}=-\frac{\sqrt{G_{v_{1}} G_{v_{4}}}}{G_{v_{2}}}$ for $v_{i} \in \Lambda(1 \leq i \leq 4)$. We

$$
A_{i} A_{i \pm 1} A_{i}=A_{i \pm 1} A_{i} A_{i \pm 1}
$$

from Lemma 1.4 and the star-triangle relation $[6,(2.2)]$. Let $P$ $=\left(\lambda^{(0)}, \lambda^{(1)}, \ldots, \lambda^{(n)}\right)$ and $Q=\left(v^{(0)}, v^{(1)}, \ldots, v^{(n)}\right)$ be two elements of $\mathscr{P}(\lambda)$ such that $\lambda^{(j)}=v^{(j)}$ for $j \in\{1,2, \ldots, n\} \backslash\{i\}$. By the definition of $A_{i}^{\prime}$, the matrix element $\left(A_{i}^{\prime}\right)_{Q P}$ is not depend on $\lambda^{(j)}, j \neq i, \quad i \pm 1$. Let $A^{\prime}\left(\lambda^{(i-1)}, \lambda^{(i)}, \nu^{(i)}, \lambda^{(i+1)}\right)$ $=\left(A_{i}^{\prime}\right)_{Q P}$. Then, from $[6,(2.12)]$ and Lemma 1.4 , we have

$$
A\left(\lambda^{(i-1)}, \lambda^{(i)}, v^{(i)}, \lambda^{(i+1)}\right)=\sqrt{\frac{G_{\lambda^{(i)}} G_{v^{(i)}}}{G_{\lambda^{(i-1)}} G_{\lambda^{(i+1)}}}} A^{\prime}\left(v^{(i)}, \lambda^{(i-1)}, \lambda^{(i+1)}, \lambda^{(i)}\right) .
$$

This formula implies the following relations. 


$$
\begin{array}{ll}
E_{i} E_{i \pm 1} A_{i}=E_{i} A_{i \pm 1}^{\prime}, & E_{i} E_{i \pm 1} A_{i}^{\prime}=E_{i} A_{i \pm 1}, \\
A_{i} E_{i \pm 1} E_{i}=A_{i \pm 1}^{\prime} E_{i}, & A_{i}^{\prime} E_{i \pm 1} E_{i}=A_{i \pm 1} E_{i} .
\end{array}
$$

From now on, we determine the eigenvalues of $A_{i}$. From (1.2b)-(1.2d), we know that $A_{i}$ has eigenvalues $q$ and $-q^{-1}$. Hence formula (1.12) implies that $A_{i}$ has at most two eigenvalues except $q$ and $-q^{-1}$. From (1.14), we know that the remaining eigenvalue is equal to $\alpha^{2} q$ or $\left(-\alpha^{2} q\right)^{-1}$. But we know that there is only one eigenvalue $\left(-\alpha^{2} q\right)^{-1}$ of $A_{i}$ except $q$ and $-q^{-1}$, since $\lim _{\alpha \rightarrow \infty} \alpha^{2} A_{i}$ is bounded. The above argument implies that $A_{i}$ acts on $\operatorname{Im} E_{i}$ by the scalar $\left(-\alpha^{2} q\right)^{-1}$. Since $E_{i}$ is a scalar multiple of a projection, we have

$$
A_{i} E_{i}=\left(-\alpha^{2} q\right)^{-1} E_{i} .
$$

The above relations (1.14)-(1.16), (1.18) and (1.19) among $A_{i}, A_{i}^{\prime}$ and $E_{i}(1 \leq i \leq n-1)$ imply that there is a representation $\rho_{\lambda}$ of $C_{n}(\alpha, q)$ with $\rho_{\lambda}\left(\tau_{i}\right)$ $=A_{i}, \rho_{\lambda}\left(\tau_{i}^{-1}\right)=A_{i}^{\prime}$ and $\rho_{\lambda}\left(\varepsilon_{i}\right)=E_{i}$. This proves the part (i) of Theorem 1.5.

From now on, we prove (ii) and (iii) inductively. We identify $C_{1}(\alpha, q)$ with $\mathbb{C}(\alpha, q)$. For $n=2, \rho_{(2)}\left(\tau_{1}\right)=q, \rho_{(11)}\left(\tau_{1}\right)=q^{-1}$ and $\rho_{\phi}\left(\tau_{1}\right)=\left(-\alpha^{2} q\right)^{-1}$. Hence the statements (ii) and (iii) are satisfied for the case $n=1,2$. Now, for $n \geq 3$, assume that $C_{n-1}(\alpha, q)$ satisfies (ii) and (iii). In the following, we identify $C_{n-1}(\alpha, q)$ with the subalgebra of $C_{n}(\alpha, q)$ generated by $\tau_{1}^{ \pm 1}, \ldots, \tau_{n-2}^{ \pm 1}$, $\varepsilon_{1}, \ldots, \varepsilon_{n-2}$. Let $\lambda \in \Lambda(n)$. Let $W_{v}$ is the subspace of $V_{v}$ spanned by the vectors $\left\{v_{\left(\mu_{0}, \mu_{1}, \ldots, \mu_{n-2}, v, \lambda\right)} \mid\left(\mu_{0}, \mu_{1}, \ldots, \mu_{n-2}, v, \lambda\right) \in \mathscr{P}(n)\right\}$. Then $W_{v}$ is $C_{n-1}(\alpha, q)$-invariant and isomorphic to $V_{v}$ as a $C_{n-1}(\alpha, q)$-module. Hence, by the induction hypothesis, $W_{v}$ is an irreducible $C_{n-1}(\alpha, q)$-module. By the definition of $V_{\lambda}$, we have $V_{\lambda}=\oplus_{\substack{v \in \Lambda(n-1) \\ v \sim \lambda}} W_{v}$. Let $U$ be a $C_{n}(\alpha, q)$-submodule of $V_{\lambda}$. Then $U$ is also a $C_{n-1}(\alpha, q)$-submodule of $V_{\lambda}$. The induction hypothesis says that $W_{v}$ and $W_{v^{\prime}}$ are not equivalent for distinct $v$ and $v^{\prime}$ in $\Lambda(n-1)$ and so $V_{\lambda}$ is multiplicity-free as an $C_{n-1}(\alpha, q)$-module. Hence, for each $v \in \Lambda(n-1)$ such that $v \sim \lambda, U \cap W_{\lambda}=W_{\lambda}$ or 0 . Now, assume that there are $v$ and $v^{\prime}$ in $\Lambda(n-1)$ such that $v \sim \lambda, v^{\prime} \sim \lambda$, $U \cap W_{v}=W_{v}$ and $U \cap W_{v}^{\prime}=0$. Lemma 1.7 shows that there is $\mu \in \Lambda(n-2)$ such that $\mu \sim v$ and $\mu \sim v^{\prime}$. Let $P=\left(p^{(o)}, p^{(1)}, \ldots, p^{(n)}\right)$ and $P^{\prime}=\left(p^{(0)}, p^{\prime(1)}, \ldots, p^{\prime(n)}\right)$ be elements of $\mathscr{P}(\lambda)$ such that $p^{(i)}=p^{\prime(i)}$ for $i \neq n-1, p^{(n-2)}=\mu, p^{(n-1)}$ $=v, p^{(n-1)}=v^{\prime}$ and $p^{(n)}=\lambda$. The construction of the representation $\rho_{\lambda}$ implies that

$$
\rho_{\lambda}\left(\tau_{n-1}\right) v_{P}=A_{P P^{\prime}} v_{P^{\prime}}+\cdots
$$

with respect to the basis $\left\{v_{Q} \mid Q \in \mathscr{P}(\lambda)\right\}$ of $V_{\lambda}$. Recall that the subspaces $W_{v}$ and $W_{v^{\prime}}$ are spanned by subsets of $\left\{v_{Q} \mid Q \in \mathscr{P}(\lambda)\right\}, \quad v_{p} \in W_{v}, v_{p^{\prime}} \in W_{v^{\prime}}$ and $A_{P P^{\prime}} \neq 0$. Since $U$ is a $C_{n}(\alpha, q)$-module, $A_{P P^{\prime}} \neq 0$ contradicts that the assumption $U \cap W_{v}^{\prime}=0$. Hence, $U$ must be $V_{\lambda}$ or 0 and so $V_{\lambda}$ is an irreducible 
$C_{n}(\alpha, q)$-module. This proves (ii).

With the induction hypothesis, Lemma 1.8 implies that $V_{\lambda_{1}}$ and $V_{\lambda_{2}}$ are not isomorphic as $C_{n-1}(\alpha, q)$-modules. Hence $V_{\lambda_{1}}$ and $V_{\lambda_{2}}$ are not isomorphic as $C_{n}(\alpha, q)$-modules. This implies (iii).

We show the last statement (iv). Since the algebra $C_{n}(\alpha, q)$ is a oneparameter deformation of the Brauer's algebra $D_{n}(\beta)$, the number of irreducible representations of $C_{n}(\alpha, q)$ is equal to that of $D_{n}(\beta)$. The number of the irreducible representations of $D_{n}(\beta)$ is given, for example, in [12], which coincides with the number of $\mathscr{P}(n)$. Hence the representations constructed above covers the all irreducible representations of $C_{n}(\alpha, q)$.

Proof of Corollary 1.6. We noted that the limit $q \rightarrow 1$ of the algebra $C_{n}\left(q^{\beta / 2}, q\right)$ is isomorphic to Brauer's algebra $D_{n}(\beta)$ in [12]. Let $\rho_{\lambda}$ be the representation of $C_{n}(\alpha, q)$ constructed above and $c(\alpha, q)$ a coefficient of the representation matrix $\rho_{\lambda}\left(\tau_{i}\right)$ of a generator $\tau_{i}(1 \leq i \leq n-1)$. Then $c\left(q^{\beta / 2}, q\right)$ has a limit with $q \rightarrow 1$ and $c\left(q^{\beta / 2}, q\right) \neq 0$ if $c(\alpha, q) \neq 0$. Hence we can apply a similar argument of the proof of Theorem 1.5 to the limiting case and we get the corollary.

\section{§ 2. The Kauffman Polynomial}

In this section we give a formula (2.1) for the Kauffman polynomial in terms of the irreducible characters of the algebra $C_{n}(\alpha, q)$ introduced in the last section. Let $\alpha$ and $q$ be indeterminates. For a positive integer $n$, let $B_{n}$ denote the braid group on $n$-strings and $\mathbf{C}(\alpha, q) B_{n}$ the group ring of $B_{n}$ over the field $\mathbf{C}(\alpha, q)$. Let $\sigma_{1}, \sigma_{2}, \ldots, \sigma_{n-1}$ be the standard generators of $B_{n}$ given as in Figure 1.

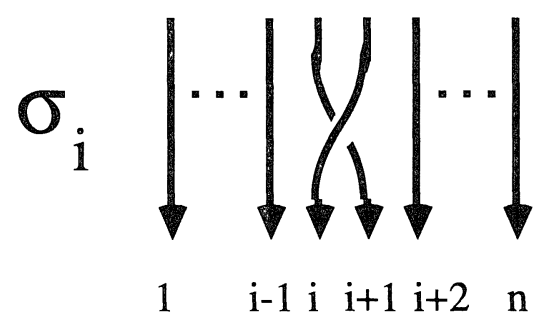

Figure 1

Let $p_{n}: \mathbf{C}(\alpha, q) B_{n} \rightarrow C_{n}(\alpha, q)$ be the algebra homomorphism defined by $p_{n}\left(\sigma_{i}\right)$ $=\left(-\alpha^{2} q\right)^{-1} \tau_{i}$. For $b \in B_{n}$, let

$$
F_{n}(b)=\left(1-\frac{\alpha^{2} q-\alpha^{-2} q^{-1}}{q-q^{-1}}\right)^{-1} \sum_{\lambda \in \Lambda(n)} G_{\lambda} \chi_{\lambda}\left(p_{n}(b)\right),
$$

where $\chi_{\lambda}$ be the character of the irreducible representation $\rho_{\lambda}$ of the algebra $C_{n}(\alpha, q)$ introduced in $\S 1$. 
Theorem 2.2. Let $b$ be an $n$-braid and $\hat{b}$ denote its closure. Let $F(\hat{b})$ $=F_{n}(b)$. Then $F$ is the Kauffman polynomial of links (see e.g. [2] and [8]).

Proof. First, we prove that $F$ is an invariant of link isotopy types. To do this, we show that $F$ is constant on a equivalence class of $B$ with respect to the Markov relation. The Markov relation $\sim$ is the equivalence relation of $B$ $=\left\{(b, n) \mid b \in B_{n}\right\}$ generated by the following.

(1) For $b_{1}, b_{2} \in B_{n},\left(b_{1} b_{2}, n\right) \sim\left(b_{2} b_{1}, n\right)$.

(2) For $b \in B_{n},(b, n) \sim\left(b \sigma_{n}^{ \pm 1}, n+1\right)$.

We have $F\left(\widehat{b_{1} b_{2}}\right)=F\left(\widehat{b_{2} b_{1}}\right)$ for $b_{1}, b_{2} \in B_{n}$ since $F_{n}$ is a linear combination of characters $\chi_{\lambda}{ }^{\circ} p_{n}$ of $B_{n}$. We show that $F(\hat{b})=F\left(\widehat{b \sigma_{n}^{ \pm 1}}\right)$ for $b \in B_{n}$. By using the construction of $\rho_{\lambda}$, the above equalities follow from Lemma 2.3 below. Let $w(b)$ denote the exponent sum of $b$. The regular isotopy invariant $L$ defined by $L(\hat{b})$ $=\left(-\alpha^{2} q\right)^{w(b)} F(\hat{b})$ satisfies the following relations.

$$
\begin{aligned}
& L\left(K_{+}\right)-L\left(K_{-}\right)=\left(q-q^{-1}\right)\left(L\left(K_{0}\right)-L\left(K_{\infty}\right)\right), \\
& L\left(K_{\ell+}\right)=-\alpha^{2} q L\left(K_{-}\right), L\left(K_{\ell-}\right)=\left(-\alpha^{2} q\right)^{-1} L\left(K_{-}\right), L(O)=1,
\end{aligned}
$$

where the $K_{*}$ are identical except within a ball where they are as in Figure 2. Hence $F$ is equal to the Kauffman polynomial by [8, Section 2].

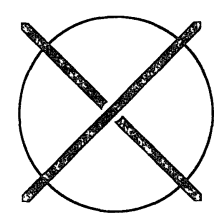

$\mathbb{K}_{+}$
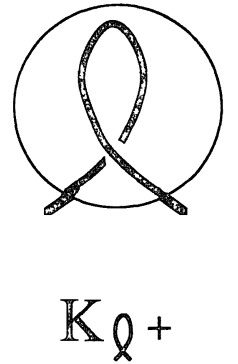
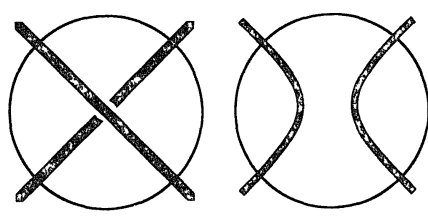

$\mathbb{K}_{0}$

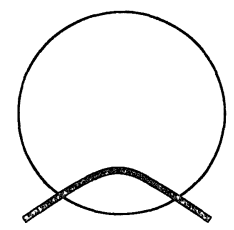

$\mathbb{K}_{n}$

Figure 2

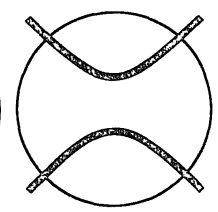

$\mathbb{K}_{\infty}$

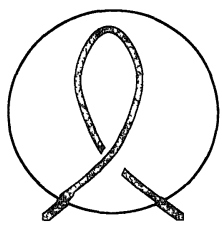

$\mathbb{K}_{\ell}-$

Lemma 2.3. For $\lambda_{1}$ and $\lambda_{2}$ in $\Lambda$ such that $\lambda_{1} \sim_{1} \lambda_{2}$, we have

$$
\sum_{\lambda_{2} \tau_{1} \lambda_{3}} \frac{G_{\lambda_{3}}}{G_{\lambda_{2}}} A\left(\lambda_{1}, \lambda_{2}, \lambda_{2}, \lambda_{3}\right)=-\alpha^{2} q .
$$


Proof. Let $\lambda_{4}$ be an element of $\Lambda$ such that $\lambda_{4} \sim_{1} \lambda_{2}$. From (1.19) and $A_{i} A_{i}^{\prime}=1$, we have $\sum_{\lambda_{2} \sim \lambda_{3}} \sqrt{G_{\lambda_{3}} G_{\lambda_{4}}} G_{\lambda_{2}}^{-1} A^{\prime}\left(\lambda_{2}, \lambda_{3}, \lambda_{1}, \lambda_{2}\right)=-\alpha^{2} q \sqrt{G_{\lambda_{1}} G_{\lambda_{4}}} G_{\lambda_{2}}^{-1}$ and so we have $\sum_{\lambda_{2} \sim \lambda_{3}} \sqrt{\frac{G_{\lambda_{3}}}{G_{\lambda_{1}}}} A^{\prime}\left(\lambda_{2}, \lambda_{3}, \lambda_{1}, \lambda_{2}\right)=-\alpha^{2} q$. Applying (1.17) to this, we get the statement of the lemma.

\section{References}

[1] Akutsu, Y. and Wadati, M., Knot invariants and the critical statistical systems, J. Phys. Soc. Japan, 56 (1987), 839-842.

[2] Akutsu, Y., Deguchi, T. and Wadati, M., Exactly solvable models and new link polynomials IV, J. Phys. Soc. Japan, 57, 1173-1185.

[3] Birman, J. S. and Wenzl, H., Braids, link polynomials and a new algebra, Trans. Amer. Math. Soc., 313 (1989), 249-273.

[4] Brauer, R., On algebras which are connected with the semisimple continuous groups, Ann. of Math., 38 (1937), 854-872.

[5] Jimbo, M., A q-difference analogue of $\mathscr{U}(\mathrm{g})$ and the Yang-Baxter equation, Lett. Math. Phys., 10 (1985), 63-69.

[6] Jimbo, M., T. Miwa and M. Okado, Solvable lattice models related to the vector representation of classical Lie algebras, Comm. Math. Phys., 116 (1988), 507-525.

[7] Lusztig, G., Singularities, character formulas, and a q-analog of weight multiplicities, Asterisque, 101-102 (1983), 208-229.

[8] Murakami, J., The Kauffman polynomial of links and representation theory, Osaka J. Math., 24 (1987), 745-758.

[9] - The parallel version of polynomial invariants of links, Osaka J. Math., 26 (1989), $1-55$.

[10] Turaev, T. G., The Yang-Baxter equation and invariants of links, Invent. Math., 92 (1988), 527-553.

[11] Wenzl, H., Hecke algebra of type $A_{n}$ and subfactors, Invent. Math., 92 (1988), 349-383.

[12] - On the structure of Brauer's centralizer algebras, Ann. of Math. (2) 128 (1988), 173-193. 
